\title{
Playing "telephone": bioactive lipids as mediators of intercompartmental communication in the alveolus
}

\author{
Marc Peters-Golden \\ Division of Pulmonary and Critical Care Medicine, The University of Michigan Health System, 6301 \\ Medical Science Research Building III, 1150 W. Medical Center Drive, Ann Arbor, Michigan 48109-0642, USA. \\ Phone: (734) 763-9077; Fax: (734) 764-4556; E-mail: petersm@umich.edu.
}

The thin alveolar wall, bordered on its airspace side by epithelium and containing a rich network of pulmonary capillaries, is ideally suited for efficient exchange of gases between the airspace and the capillary vasculature. However, the lung was engineered with additional imperatives in mind. The presence of an inflammatory stimulus in any tissue must be communicated to the local vasculature so that circulating leukocytes can be recruited, and nowhere is this more crucial than in the lungs. With a surface area approximating that of a tennis court, the lungs represent the body's largest internal interface with the outside world. Moreover, the airspaces are continually assaulted by a barrage of inhaled and aspirated microorganisms, antigens, and irritants. When local defense mechanisms (e.g., phagocytosis by resident alveolar macrophages) are overwhelmed, recruited leukocytes are needed to contain the insult. Yet the inflammatory response that ensues must be confined to the affected alveoli in order to minimize any impairment of gas exchange.

This need for communication between airspaces and vascular compartments requires epithelial and endothelial cells in the alveolar wall to sense and respond to changes in their local milieu. Indeed, research over the last 30 years has begun to elucidate the vast repertoire of receptors and intracellular signaling systems used by these 2 cell types to carry out this mission (1, 2). However, these cells must also be able to transmit information to each other. The nature of the currency exchanged in such intercellular trans-

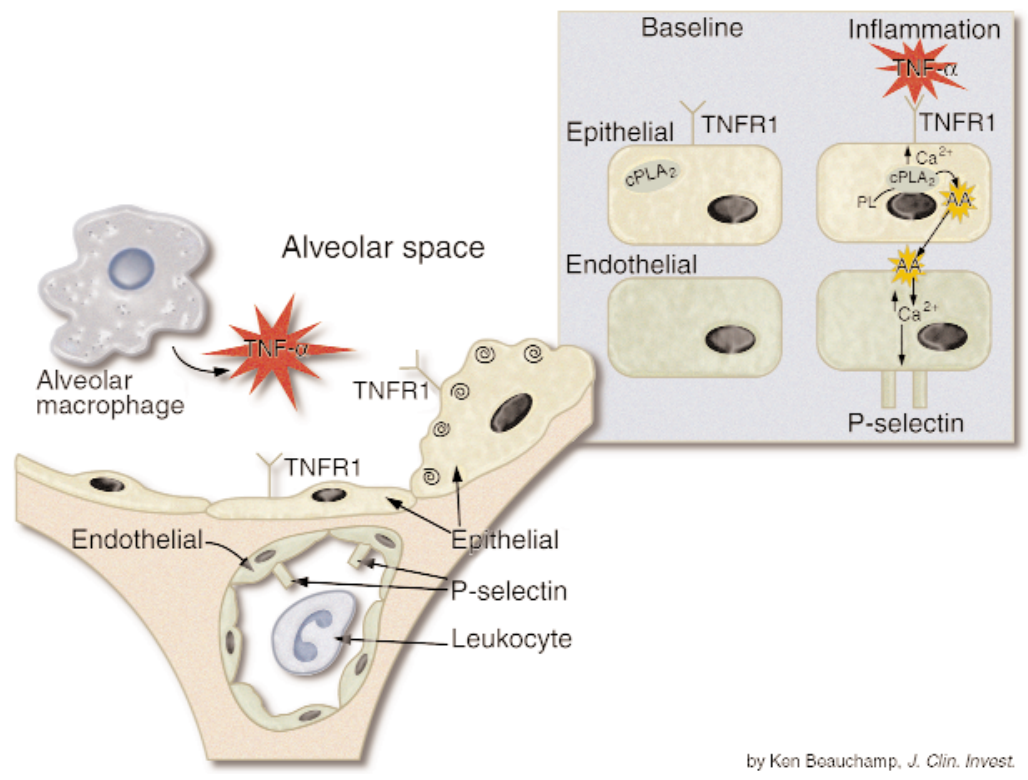

Figure 1

Scheme for alveolar epithelial to endothelial cell signal propagation initiated by TNF- $\alpha$. The spatial interrelationships of participating cells and receptors are shown on the left. The molecular events are summarized on the right. The crucial molecule mediating intercellular communication is free AA, which is hydrolyzed from epithelial cell nuclear membrane phospholipids (PL) by cPLA, and which induces endothelial cell $\mathrm{Ca}^{2+}$ flux. actions has been more difficult to pinpoint. Although in vitro experiments using cocultures of alveolar cells provide useful information about cell-cell communication (3), studies are needed in the intact lung, where boundaries between compartments are preserved.

In a series of elegant experiments published in this issue of the JCI, Kuebler and coworkers have delineated the molecular events that account for propagation of inflammatory signals from alveolar epithelial cells to endothelial cells (4). The requirement for fidelity of signal transmission at each of the many steps along this sequence is reminiscent of that required in the childhood game of "telephone." The authors have modeled alveolar inflammation by microinjecting the proinflammatory cytokine TNF- $\alpha$ into the airspace of the isolated, ventilated, and perfused rat lung. Using in situ fluorescence microscopy to quantitate intracellular $\mathrm{Ca}^{2+}$, they demonstrated that TNF- $\alpha$ elicits an increase in epithelial cell $\mathrm{Ca}^{2+}$ in a manner that depends on ligation of its surface receptor, TNFR1. The authors also observed parallel increases in endothelial $\mathrm{Ca}^{2+}$, and showed that expression of the adhesion molecule P-selectin increases on the surface of endothelial cells, a response that would be expected to initiate leukocyte recruitment. The most crucial experiments, however, were those that elucidated the nature of the signal that was released from the basolateral surface of the epithelium, causing endothelial $\mathrm{Ca}^{2+}$ to increase.

That signal, as Kuebler et al. showed conclusively, is arachidonic acid (AA). AA is a 20 -carbon unsaturated fatty acid that is esterified in cell membrane phospholipids. It is enzymatically cleaved from the parent phospholipid by the action of 1 or more of a family of enzymes termed phospholipases $\mathrm{A}_{2}$ $\left(\mathrm{PLA}_{2}\right)(5)$. Once liberated, AA is available to be metabolized through several dis- 
crete pathways to an array of bioactive substances known as eicosanoids. Among these products, the prostanoids and leukotrienes have been the most extensively investigated (6). Studies with isolated and cultured alveolar epithelial cells have demonstrated that these cells can liberate AA in response to agonists that increase intracellular $\mathrm{Ca}^{2+}$, and can metabolize this lipid, primarily to prostanoids (7). The predominant $\mathrm{PLA}_{2}$ isoform in these cells appears to be an $85-\mathrm{kDa}$ species known as cytosolic $\mathrm{PLA}_{2}$ $\left(\mathrm{cPLA}_{2}\right)(8)$. Interestingly, $\mathrm{CPLA}_{2}$ is active only after it has been translocated, in a $\mathrm{Ca}^{2+}$-dependent step, from cytosol to the nuclear envelope, where phospholipid hydrolysis takes place (9). In their in situ immunofluorescence microscopy studies, Kuebler et al. took advantage of this fact and inferred cPLA ${ }_{2}$ activation from its perinuclear locale. They further confirmed the importance of this enzyme in propagating the epithelial to endothelial signal by showing that endothelial $\mathrm{Ca}^{2+}$ entry could be prevented by alveolar infusion of either $\mathrm{Ca}^{2+}$ chelators or specific inhibitors of $\mathrm{CPLA}_{2}$.

Many readers will be surprised by the finding that $\mathrm{AA}$, rather than one of its better-known eicosanoid metabolites, proved to be the mediator of cell-cell communication in this model. However, the ability of exogenous FFAs to mimic the actions of TNF- $\alpha$ and the failure of inhibitors of AA metabolism to abrogate the endothelial response to the cytokine both indicate that AA itself represents the transmitted signal. Moreover, a growing body of literature has demonstrated that free AA, and often other unsaturated fatty acids as well, are indeed pluripotent effectors of signal transduction. Thus, AA has been shown not only to increase $\mathrm{Ca}^{2+}$ flux (10), as it did in alveolar capillary endothelial cells in the study by Kuebler et al., but also to promote superoxide generation (11), to activate kinases including protein kinase C (12) and mitogen-activated protein kinase (13), to induce expression of heat-shock genes (14), to act as a ligand for the peroxisome proliferator-activated receptor family of transcription factors (15), and to participate in the activation of nuclear factor- $\kappa \mathrm{B}(16)$.

Is AA a logical choice to mediate intercellular communication in vivo? Ideally, such a mediator would (a) be produced rapidly but transiently; (b) diffuse readily out of the source cell and into the recipient cell; (c) rapidly elicit an effector signal in the recipient cell; and (d) have a relatively short half-life. These features would facilitate propagation of a message that is quick, short-lived, and local. A small amphipathic molecule that can be generated within seconds to minutes by virtually all cell types, AA readily meets these criteria. The modulation of $\mathrm{CPLA}_{2}$ expression and enzymatic activity (17) provides further opportunities for fine-tuning this signal. By contrast, many proteins, such as cytokines, are ill suited to act as rapid-response signals by virtue of their size and the temporal requirement for transcriptional or translational induction.

A number of key issues remain to be addressed. First, it must be acknowledged that the experimental system under investigation in the article by Kuebler and colleagues is only a model for pulmonary inflammation. It will be important to test the participation of this molecular "telephone chain," or variants thereof, in studies using physiologically relevant inflammatory agents, such as microbes. Second, the cellular sensors for FFAs are, in many instances, not known. The identity of such sensors and the mechanisms by which they couple to signaling cascades will be an important subject for future research. Third, the potential of other candidate lipid mediators of intercompartmental communication must be explored; these include other fatty acids, other products of $\mathrm{PLA}_{2}$ action (lysophospholipids and platelet activating factor), and eicosanoids.

There is 1 final implication of this work for those willing to indulge in symbolism. An important feature of the experimental model dissected by Kuebler and colleagues is the cooperation between a cytokine and a lipid. I am often struck by the manner in which scientists studying either cytokines or lipids themselves appear to be segregated into 2 parallel uni- verses. Scientific inquiry would surely be advanced if communication between investigators inhabiting these 2 worlds occurred as readily as it certainly does between these 2 classes of mediators in biological systems.

1. Pober, J. 1999. Immnunobiology of human vascular endothelium. Immunol. Res. 19:225-232.

2. Simon, R., and Paine, R.R. 1995. Participation of pulmonary alveolar epithelial cells in lung inflammation. J. Lab. Clin. Med. 126:108-118.

3. Gueven, N., Glatthaar, B., Manke, H., and Haemmerle, H. 1996. Co-cultivation of rat pneumocytes and bovine endothelial cells on a liquid interface. Eur. Respir. J. 9:968-975.

4. Kuebler, W., Parthasarathi, K., Wang, P., and Bhattacharya, J. 2000. A novel signaling mechanism between gas and blood compartments of the lung. J. Clin. Invest. 105:905-913 (2000).

5. Dennis, E. 1997. The growing phospholipase $A_{2}$ superfamily of signal transduction enzymes. Trends Biochem. Sci. 22:1-2.

6. Serhan, C., Haeggstrom, J., and Leslie, C. 1996. Lipid mediator networks in cell signaling: update and impact of cytokines. FASEB J. 10:1147-1158.

7. Chauncey, J., Peters-Golden, M., and Simon, R. 1988. Arachidonic acid metabolism by rat alveolar epithelial cells. Lab. Invest. 58:133-140.

8. Neagos, G., Feyssa, A., and Peters-Golden, M. 1993. Phospholipase $A_{2}$ in alveolar type II epithelial cells: biochemical and immunologic characterization. Am. J. Physiol. Lung Cell. Mol. Physiol. 264:L261-L268.

9. Peters-Golden, M., Song, K., Marshall, T., and Brock, T. 1996. Translocation of cytosolic phospholipase $A_{2}$ to the nuclear envelope elicits topographically localized phospholipid hydrolysis. Biochem. J. 318:797-803.

10. Kolesnick, R., Musacchio, I., Thaw, C., and Gershengorn, M. 1984. Arachidonic acid mobilizes calcium and stimulates prolactin secretion from GH3 cells. Am. J. Physiol. Endocrinol. Metab. 246:E458-E462.

11. Badwey, J., Curnutte, J., and Karnovsky, M. 1981. cis-polyunsaturated fatty acids induce high levels of superoxide production by human neutrophils. J. Biol. Chem. 256:12640-12643.

12. Murakami, K., and Routtenberg, A. 1985. Direct activation of purified protein kinase $\mathrm{C}$ by unsaturated fatty acids (oleate and arachidonate) in the absence of phospholipids and Ca2+. FEBS Lett. 192:189-193.

13. Rao, G., et al. 1994. Activation of mitogen-activated protein kinases by arachidonic acid and its metabolites in vascular smooth muscle cells. $J$. Biol. Chem. 269:32586-32591.

14. Jurivich, D., Sistonen, L., Sarge, K., and Morimoto, R. 1994. Arachidonate is a potent modulator of human heat shock gene transcription. Proc. Natl. Acad. Sci. USA. 91:2280-2284.

15. Schmidt, A., et al. 1992. Identification of a new member of the steroid hormone receptor superfamily that is activated by a peroxisome proliferator and fatty acids. Mol. Endocrinol. 6:1634-1641.

16. van Puijenbroek, A., Wissink, S., van der Saag, P., and Peppelenbosch, M. 1999. Phospholipase $A_{2}$ inhibitors and leukotriene synthesis inhibitors block TNF-induced NF- $\mathrm{KB}$ activation. Cytokine. 11:104-110.

17. Gijon, M., and Leslie, C. 1999. Regulation of arachidonic acid release and cytosolic phospholipase $\mathrm{A}_{2}$ activation. J. Lenkoc. Biol. 65:330-336. 